\title{
Algebra of Polynomially Bounded Sequences and Negligible Functions
}

\author{
Hiroyuki Okazaki \\ Shinshu University \\ Nagano, Japan
}

\begin{abstract}
Summary. In this article we formalize negligible functions that play an essential role in cryptology [10, 2]. Generally, a cryptosystem is secure if the probability of succeeding any attacks against the cryptosystem is negligible. First, we formalize the algebra of polynomially bounded sequences [20. Next, we formalize negligible functions and prove the set of negligible functions is a subset of the algebra of polynomially bounded sequences. Moreover, we then introduce equivalence relation between polynomially bounded sequences, using negligible functions.
\end{abstract}

MSC: 68Q25 94A60 03B35

Keywords: polynomially bounded function; negligible functions

MML identifier: ASYMPT_3, version: 8.1.04 5.33.1254

The notation and terminology used in this paper have been introduced in the following articles: [29], [16], [17, [20], [4], 19], [9], 24], 21], [5], 6], 26], 25], [1], [7], [13], [22], [12], 3], [11], [30], [27], 14], [15], 23], 28], [18], and [8].

\section{Preliminaries}

Let us consider a real number $r$. Now we state the propositions:

(1) $r<|r|+1$.

(2) There exists a natural number $N$ such that for every natural number $n$ such that $N \leqslant n$ holds $r<\frac{n}{\log _{2} n}$.

Let us consider a natural number $k$. Now we state the propositions:

(3) There exists a natural number $N$ such that for every natural number $x$ such that $N \leqslant x$ holds $x^{k}<2^{x}$. The theorem is a consequence of (2). 
(4) There exists a natural number $N$ such that for every natural number $x$ such that $N \leqslant x$ holds $\frac{1}{2^{x}}<\frac{1}{x^{k}}$. The theorem is a consequence of (3).

Now we state the proposition:

(5) Let us consider a natural number $z$. Suppose $2 \leqslant z$. Let us consider a natural number $k$. Then there exists a natural number $N$ such that for every natural number $x$ such that $N \leqslant x$ holds $\frac{1}{z^{x}}<\frac{1}{x^{k}}$. The theorem is a consequence of (4).

Observe that there exists a finite 0 -sequence of $\mathbb{R}$ which is positive yielding and there exists a positive yielding finite 0 -sequence of $\mathbb{R}$ which is non empty.

Now we state the proposition:

(6) Let us consider a finite 0 -sequence $c$ of $\mathbb{R}$, and a real number $a$. Then $a \cdot c$ is a finite 0 -sequence of $\mathbb{R}$.

Let $c$ be a finite 0 -sequence of $\mathbb{R}$ and $a$ be a real number. Observe that $a \cdot c$ is finite as a transfinite sequence of elements of $\mathbb{R}$.

Now we state the proposition:

(7) Let us consider a non empty, positive yielding finite 0 -sequence $c$ of $\mathbb{R}$, and a real number $a$. Suppose $0<a$. Then $a \cdot c$ is a non empty, positive yielding finite 0 -sequence of $\mathbb{R}$. The theorem is a consequence of (6).

Let $c$ be a non empty, positive yielding finite 0 -sequence of $\mathbb{R}$ and $a$ be a positive real number. Observe that $a \cdot c$ is non empty and positive yielding as a finite 0 -sequence of $\mathbb{R}$.

Let $c$ be a finite 0 -sequence of $\mathbb{R}$. We introduce the notation polynom $c$ as a synonym of $\operatorname{Seq}_{\text {poly }}(c)$.

Now we state the propositions:

(8) Let us consider a non empty, positive yielding finite 0 -sequence $c$ of $\mathbb{R}$, and a natural number $x$. Then $0<(\operatorname{polynom} c)(x)$.

Proof: Define $\mathcal{P}$ [natural number] $\equiv$ for every non empty, positive yielding finite 0 -sequence $c$ of $\mathbb{R}$ such that len $c=\$_{1}$ for every natural number $x$, $0<($ polynom $c)(x)$. For every natural number $k$ such that $\mathcal{P}[k]$ holds $\mathcal{P}[k+1]$ by [20, (28), (29)], [1, (44)], [5, (3), (47)]. For every natural number $k, \mathcal{P}[k]$ from [1, Sch. 2].

(9) Let us consider non empty, positive yielding finite 0 -sequences $c, c_{1}$ of $\mathbb{R}$, and a real number $a$. Suppose $c_{1}=a \cdot c$. Let us consider a natural number $x$. Then $\left(\right.$ polynom $\left.c_{1}\right)(x)=a \cdot($ polynom $c)(x)$.

Proof: For every object $i$ such that $i \in \operatorname{dom}\left(c_{1} \cdot\left\{x^{1 \cdot n+0}\right\}_{n \in \mathbb{N}}\right)$ holds $\left(c_{1} \cdot\left\{x^{1 \cdot n+0}\right\}_{n \in \mathbb{N}}\right)(i)=\left(a \cdot\left(c \cdot\left\{x^{1 \cdot n+0}\right\}_{n \in \mathbb{N}}\right)\right)(i)$ by [20, $\left.(26)\right]$. 


\section{Algebra of Polynomially Bounded Sequences}

Let $p$ be a sequence of real numbers. We say that $p$ is absolutely polynomially bounded if and only if

(Def. 1) there exists a natural number $k$ such that $|p| \in O\left(\left\{n^{k}\right\}_{n \in \mathbb{N}}\right)$.

One can verify that every sequence of real numbers which is polynomially bounded is also absolutely polynomially bounded.

Now we state the proposition:

(10) Let us consider an element $r$ of $\mathbb{N}$, and a sequence $s$ of real numbers. If $s=\mathbb{N} \longmapsto r$, then $s$ is absolutely polynomially bounded.

One can check that there exists a function from $\mathbb{N}$ into $\mathbb{R}$ which is absolutely polynomially bounded.

Let $f, g$ be absolutely polynomially bounded functions from $\mathbb{N}$ into $\mathbb{R}$. One can verify that $f+g$ is absolutely polynomially bounded as a function from $\mathbb{N}$ into $\mathbb{R}$ and $f \cdot g$ is absolutely polynomially bounded as a function from $\mathbb{N}$ into $\mathbb{R}$.

Let $f$ be an absolutely polynomially bounded function from $\mathbb{N}$ into $\mathbb{R}$ and $a$ be an element of $\mathbb{R}$. Observe that $a \cdot f$ is absolutely polynomially bounded as a function from $\mathbb{N}$ into $\mathbb{R}$.

The functor $\mathcal{O}_{\text {poly }}$ yielding a subset of RAlgebra $\mathbb{N}$ is defined by

(Def. 2) for every object $x, x \in$ it iff $x$ is an absolutely polynomially bounded function from $\mathbb{N}$ into $\mathbb{R}$.

Note that $\mathcal{O}_{\text {poly }}$ is non empty.

The functor RAlgebra $\mathcal{O}_{\text {poly }}$ yielding a strict algebra structure is defined by

(Def. 3) the carrier of it $=\mathcal{O}_{\text {poly }}$ and the multiplication of $i t=\mathbb{R}^{\mathbb{N}}\left\lceil\mathcal{O}_{\text {poly }}\right.$ and the addition of $i t=+_{\mathbb{R}^{\mathbb{N}}} \uparrow \mathcal{O}_{\text {poly }}$ and the external multiplication of $i t=$ $\cdot \mathbb{R}_{\mathbb{R}^{\mathbb{N}}} \uparrow\left(\mathbb{R} \times \mathcal{O}_{\text {poly }}\right)$ and the one of $i t=\mathbf{1}_{\mathbb{R}^{\mathbb{N}}}$ and the zero of $i t=\mathbf{0}_{\mathbb{R}^{\mathbb{N}}}$.

One can verify that $\mathrm{RAlgebra} \mathcal{O}_{\text {poly }}$ is non empty.

Now we state the propositions:

(11) The carrier of RAlgebra $\mathcal{O}_{\text {poly }} \subseteq$ the carrier of RAlgebra $\mathbb{N}$.

(12) Let us consider an object $f$. Then $f \in \mathrm{RAlgebra} \mathcal{O}_{\text {poly }}$ if and only if $f$ is an absolutely polynomially bounded function from $\mathbb{N}$ into $\mathbb{R}$.

Let us consider points $f, g$ of RAlgebra $\mathcal{O}_{\text {poly }}$ and points $f_{1}, g_{1}$ of RAlgebra $\mathbb{N}$.

Let us assume that $f=f_{1}$ and $g=g_{1}$. Now we state the propositions:

(13) $f \cdot g=f_{1} \cdot g_{1}$.

(14) $f+g=f_{1}+g_{1}$.

Now we state the propositions: 
(15) Let us consider a point $f$ of RAlgebra $\mathcal{O}_{\text {poly }}$, a point $f_{1}$ of RAlgebra $\mathbb{N}$, and an element $a$ of $\mathbb{R}$. If $f=f_{1}$, then $a \cdot f=a \cdot f_{1}$.

(16) $0_{\text {RAlgebra }} \mathcal{O}_{\text {poly }}=0_{\text {RAlgebra } \mathbb{N}}$.

(17) $1_{\text {RAlgebra } \mathcal{O}_{\text {poly }}}=1_{\text {RAlgebra } \mathbb{N}}$.

One can check that RAlgebra $\mathcal{O}_{\text {poly }}$ is strict, Abelian, add-associative, right zeroed, right complementable, commutative, associative, right unital, right distributive, vector associative, scalar associative, vector distributive, and scalar distributive.

Now we state the proposition:

(18) RAlgebra $\mathcal{O}_{\text {poly }}$ is an algebra.

Let us consider vectors $f, g, h$ of RAlgebra $\mathcal{O}_{\text {poly }}$ and functions $f^{\prime}, g^{\prime}, h^{\prime}$ from $\mathbb{N}$ into $\mathbb{R}$.

Let us assume that $f^{\prime}=f$ and $g^{\prime}=g$ and $h^{\prime}=h$. Now we state the propositions:

(19) $h=f+g$ if and only if for every natural number $x, h^{\prime}(x)=f^{\prime}(x)+g^{\prime}(x)$. The theorem is a consequence of (11) and (14).

(20) $h=f \cdot g$ if and only if for every natural number $x, h^{\prime}(x)=f^{\prime}(x) \cdot g^{\prime}(x)$. The theorem is a consequence of (11) and (13).

Now we state the proposition:

(21) Let us consider vectors $f, h$ of RAlgebra $\mathcal{O}_{\text {poly }}$, and functions $f^{\prime}, h^{\prime}$ from $\mathbb{N}$ into $\mathbb{R}$. Suppose $f^{\prime}=f$ and $h^{\prime}=h$. Let us consider a real number $a$. Then $h=a \cdot f$ if and only if for every natural number $x, h^{\prime}(x)=a \cdot f^{\prime}(x)$. The theorem is a consequence of (11) and (15).

\section{Negligible Functions}

Definition 1.3.5 of [10], P.16: Let $f$ be a function from $\mathbb{N}$ into $\mathbb{R}$. We say that $f$ is negligible if and only if

(Def. 4) for every non empty, positive yielding finite 0-sequence $c$ of $\mathbb{R}$, there exists a natural number $N$ such that for every natural number $x$ such that $N \leqslant x$ holds $|f(x)|<\frac{1}{(\operatorname{polynom} c)(x)}$.

Now we state the propositions:

(22) Let us consider a real number $r$. Suppose $0<r$. Then there exists a non empty, positive yielding finite 0 -sequence $c$ of $\mathbb{R}$ such that for every natural number $x$, $($ polynom $c)(x)=r$.

(23) Let us consider a function $f$ from $\mathbb{N}$ into $\mathbb{R}$. Suppose $f$ is negligible. Let us consider a real number $r$. Suppose $0<r$. Then there exists a natural 
number $N$ such that for every natural number $x$ such that $N \leqslant x$ holds $|f(x)|<r$. The theorem is a consequence of $(22)$.

(24) Let us consider a function $f$ from $\mathbb{N}$ into $\mathbb{R}$. If $f$ is negligible, then $f$ is convergent and $\lim f=0$. The theorem is a consequence of (23).

Let us observe that $\{0\}_{n \in \mathbb{N}}$ is negligible and there exists a function from $\mathbb{N}$ into $\mathbb{R}$ which is negligible.

Let $f$ be a negligible function from $\mathbb{N}$ into $\mathbb{R}$. Let us observe that $|f|$ is negligible as a function from $\mathbb{N}$ into $\mathbb{R}$.

Let $a$ be a real number. One can verify that $a \cdot f$ is negligible as a function from $\mathbb{N}$ into $\mathbb{R}$.

Let $f, g$ be negligible functions from $\mathbb{N}$ into $\mathbb{R}$. One can check that $f+g$ is negligible as a function from $\mathbb{N}$ into $\mathbb{R}$ and $f \cdot g$ is negligible as a function from $\mathbb{N}$ into $\mathbb{R}$.

Now we state the propositions:

(25) InVERse OF POWER OF 2 IS NEGLIGIBLE:

Let us consider a function $f$ from $\mathbb{N}$ into $\mathbb{R}$. If for every natural number $x, f(x)=\frac{1}{2^{x}}$, then $f$ is negligible.

Proof: Set $k=$ len $c$. Define $\mathcal{F}$ (natural number) $=1 \cdot \$_{1}^{k}$. Consider $y$ being a sequence of real numbers such that for every natural number $x, y(x)=$ $\mathcal{F}(x)$ from [14, Sch. 1]. Consider $N_{1}$ being a natural number such that for every natural number $x$ such that $N_{1} \leqslant x$ holds $\left|\left(\operatorname{Seq}_{\text {poly }}(c)\right)(x)\right| \leqslant y(x)$. Consider $N_{2}$ being a natural number such that for every natural number $x$ such that $N_{2} \leqslant x$ holds $\frac{1}{2^{x}}<\frac{1}{x^{k}}$. Set $N=N_{1}+N_{2}$. For every natural number $x$ such that $N \leqslant x$ holds $|f(x)|<\frac{1}{(\text { polynom } c)(x)}$ by [1, (12)], (8).

(26) Let us consider functions $f, g$ from $\mathbb{N}$ into $\mathbb{R}$. Suppose $f$ is negligible and for every natural number $x,|g(x)| \leqslant|f(x)|$. Then $g$ is negligible.

One can check that every function from $\mathbb{N}$ into $\mathbb{R}$ which is negligible is also absolutely polynomially bounded.

The functor negligible-Funcs yielding a subset of $\mathcal{O}_{\text {poly }}$ is defined by

(Def. 5) for every object $x, x \in$ it iff $x$ is a negligible function from $\mathbb{N}$ into $\mathbb{R}$.

Let us observe that negligible-Funcs is non empty.

Let us consider vectors $v, w$ of RAlgebra $\mathcal{O}_{\text {poly }}$ and functions $v_{1}, w_{1}$ from $\mathbb{N}$ into $\mathbb{R}$.

Let us assume that $v=v_{1}$ and $w_{1}=w$. Now we state the propositions:

(27) $v+w=v_{1}+w_{1}$. The theorem is a consequence of (19).

(28) $v \cdot w=v_{1} \cdot w_{1}$. The theorem is a consequence of (20).

Now we state the propositions: 
(29) Let us consider a real number $a$, a vector $v$ of RAlgebra $\mathcal{O}_{\text {poly }}$, and a function $v_{1}$ from $\mathbb{N}$ into $\mathbb{R}$. If $v=v_{1}$, then $a \cdot v=a \cdot v_{1}$. The theorem is a consequence of (21).

(30) Let us consider a real number $a$, and a vector $v$ of RAlgebra $\mathcal{O}_{\text {poly }}$. Suppose $v \in$ negligible-Funcs. Then $a \cdot v \in$ negligible-Funcs. The theorem is a consequence of (29).

Let us consider vectors $v, u$ of RAlgebra $\mathcal{O}_{\text {poly }}$.

Let us assume that $v, u \in$ negligible-Funcs. Now we state the propositions:

(31) $v+u \in$ negligible-Funcs. The theorem is a consequence of (27).

(32) $v \cdot u \in$ negligible-Funcs. The theorem is a consequence of (28).

Let $f, g$ be functions from $\mathbb{N}$ into $\mathbb{R}$. We say that $f \approx_{\text {neg }} g$ if and only if

(Def. 6) there exists a function $h$ from $\mathbb{N}$ into $\mathbb{R}$ such that $h$ is negligible and for every natural number $x,|f(x)-g(x)| \leqslant|h(x)|$.

One can verify that the predicate is reflexive and symmetric.

Now we state the propositions:

(33) Let us consider functions $f, g, h$ from $\mathbb{N}$ into $\mathbb{R}$. Suppose $f \approx_{\text {neg }} g$ and $g \approx_{\text {neg }} h$. Then $f \approx_{\text {neg }} h$.

(34) Let us consider functions $f, g$ from $\mathbb{N}$ into $\mathbb{R}$. Then $f \approx_{\text {neg }} g$ if and only if $f-g$ is negligible. The theorem is a consequence of (26).

(35) Let us consider a non empty, positive yielding finite 0 -sequence $c$ of $\mathbb{R}$. Then there exists a real number $a$ and there exist natural numbers $k, N$ such that $0<a$ and $0<k$ and for every natural number $x$ such that $N \leqslant x$ holds (polynom $c$ ) $(x) \leqslant a \cdot x^{k}$. The theorem is a consequence of (8).

Let $a$ be a non-negative yielding finite 0 -sequence of $\mathbb{R}$ and $b$ be a nonnegative yielding sequence of real numbers. Let us observe that $a \cdot b$ is nonnegative yielding.

Let $a, b$ be non-negative yielding finite 0 -sequences of $\mathbb{R}$. One can check that $a^{\frown} b$ is non-negative yielding.

Let $a, b, c$ be non negative real numbers. Let us note that $\left\{a^{b \cdot n+c}\right\}_{n \in \mathbb{N}}$ is non-negative yielding.

Now we state the propositions:

(36) Let us consider a real number $a$, and a natural number $k$. Then there exists a non empty, positive yielding finite 0 -sequence $c$ of $\mathbb{R}$ such that for every natural number $x, a \cdot x^{k} \leqslant(\operatorname{polynom} c)(x)$.

Proof: Reconsider $c=\mathbb{Z}_{k+1} \longmapsto|a|+1$ as a finite 0 -sequence of $\mathbb{R}$. For every natural number $x, a \cdot x^{k} \leqslant(\operatorname{polynom} c)(x)$ by [14, (1)], 24, (13), (7)], [1, (44)]. 
(37) Let us consider non empty, positive yielding finite 0 -sequences $c, s$ of $\mathbb{R}$. Then there exists a non empty, positive yielding finite 0 -sequence $d$ of $\mathbb{R}$ and there exists a natural number $N$ such that for every natural number $x$ such that $N \leqslant x$ holds $(\operatorname{polynom} c)(x) \cdot(\operatorname{polynom} s)(x) \leqslant(\operatorname{polynom} d)(x)$. Proof: Consider $a_{1}$ being a real number, $k_{1}, N_{1}$ being natural numbers such that $0<a_{1}$ and $0<k_{1}$ and for every natural number $x$ such that $N_{1} \leqslant x$ holds (polynom $\left.c\right)(x) \leqslant a_{1} \cdot x^{k_{1}}$. Consider $a_{2}$ being a real number, $k_{2}, N_{2}$ being natural numbers such that $0<a_{2}$ and $0<k_{2}$ and for every natural number $x$ such that $N_{2} \leqslant x$ holds (polynom $\left.s\right)(x) \leqslant a_{2} \cdot x^{k_{2}}$. Consider $d$ being a non empty, positive yielding finite 0 -sequence of $\mathbb{R}$ such that for every natural number $x, a_{1} \cdot a_{2} \cdot x^{k_{1}+k_{2}} \leqslant(\operatorname{polynom} d)(x)$. $0<($ polynom $c)(x) .0<($ polynom $s)(x) \cdot a_{1} \cdot x^{k_{1}} \cdot\left(a_{2} \cdot x^{k_{2}}\right)=\left(a_{1} \cdot a_{2}\right) \cdot x^{k_{1}+k_{2}}$ by $[22,(27)]$.

Let $f$ be a negligible function from $\mathbb{N}$ into $\mathbb{R}$ and $c$ be a non empty, positive yielding finite 0 -sequence of $\mathbb{R}$. Let us observe that polynom $c \cdot f$ is negligible as a function from $\mathbb{N}$ into $\mathbb{R}$.

Now we state the proposition:

(38) Let us consider an absolutely polynomially bounded function $g$ from $\mathbb{N}$ into $\mathbb{R}$. Then there exists a non empty, positive yielding finite 0 -sequence $d$ of $\mathbb{R}$ and there exists a natural number $N$ such that for every natural number $x$ such that $N \leqslant x$ holds $|g(x)| \leqslant($ polynom $d)(x)$. The theorem is a consequence of (36).

Let $f$ be a negligible function from $\mathbb{N}$ into $\mathbb{R}$ and $g$ be an absolutely polynomially bounded function from $\mathbb{N}$ into $\mathbb{R}$. Let us note that $g \cdot f$ is negligible as a function from $\mathbb{N}$ into $\mathbb{R}$.

Now we state the proposition:

(39) Let us consider vectors $v, w$ of RAlgebra $\mathcal{O}_{\text {poly }}$.

Suppose $w \in$ negligible-Funcs. Then $v \cdot w \in$ negligible-Funcs. The theorem is a consequence of (12) and (28).

ACKNOWLEDGEMENT: The author would like to express his gratitude to Prof. Yuichi Futa and Prof. Yasunari Shidama for their support and encouragement.

\section{REFERENCES}

[1] Grzegorz Bancerek. The fundamental properties of natural numbers Formalized Mathematics, 1(1):41-46, 1990.

[2] Mihir Bellare. A note on negligible functions, 2002.

[3] Józef Białas. Group and field definitions. Formalized Mathematics, 1(3):433-439, 1990.

[4] Czesław Byliński. The complex numbers. Formalized Mathematics, 1(3):507-513, 1990. 
[5] Czesław Byliński. Functions and their basic properties Formalized Mathematics, 1(1): 55-65, 1990.

[6] Czesław Byliński. Functions from a set to a set Formalized Mathematics, 1(1):153-164, 1990.

[7] Czesław Byliński. Partial functions. Formalized Mathematics, 1(2):357-367, 1990.

[8] Czesław Byliński. Some basic properties of sets Formalized Mathematics, 1(1):47-53, 1990.

[9] Agata Darmochwał. Finite sets. Formalized Mathematics, 1(1):165-167, 1990.

[10] Oded Goldreich. Foundations of Cryptography: Volume 1, Basic Tools. Cambridge University Press, 2001.

[11] Krzysztof Hryniewiecki. Basic properties of real numbers Formalized Mathematics, 1(1): 35-40, 1990.

[12] Andrzej Kondracki. Basic properties of rational numbers Formalized Mathematics, 1(5): 841-845, 1990.

[13] Artur Korniłowicz. On the real valued functions. Formalized Mathematics, 13(1):181-187, 2005.

[14] Jarosław Kotowicz. Real sequences and basic operations on them. Formalized Mathematics, 1(2):269-272, 1990.

[15] Jarosław Kotowicz. Convergent sequences and the limit of sequences Formalized Mathematics, 1(2):273-275, 1990.

[16] Richard Krueger, Piotr Rudnicki, and Paul Shelley. Asymptotic notation. Part I: Theory Formalized Mathematics, 9(1):135-142, 2001.

[17] Richard Krueger, Piotr Rudnicki, and Paul Shelley. Asymptotic notation. Part II: Examples and problems. Formalized Mathematics, 9(1):143-154, 2001.

[18] Eugeniusz Kusak, Wojciech Leończuk, and Michał Muzalewski. Abelian groups, fields and vector spaces. Formalized Mathematics, 1(2):335-342, 1990.

[19] Adam Naumowicz. Conjugate sequences, bounded complex sequences and convergent complex sequences. Formalızed Mathematıcs, 6(2):265-268, 1997.

[20] Hiroyuki Okazaki and Yuichi Futa. Polynomially bounded sequences and polynomial sequences. Formalized Mathematics, 23(3):205-213, 2015. doi:10.1515/forma-2015-0017.

[21] Henryk Oryszczyszyn and Krzysztof Prażmowski. Real functions spaces. Formalized Mathematics, 1(3):555-561, 1990.

[22] Konrad Raczkowski and Andrzej Nędzusiak. Real exponents and logarithms Formalized Mathematics, 2(2):213-216, 1991.

[23] Konrad Raczkowski and Andrzej Nędzusiak. Series. Formalized Mathematics, 2(4):449452, 1991.

[24] Andrzej Trybulec. Binary operations applied to functions Formalized Mathematics, 1 (2):329-334, 1990.

[25] Michał J. Trybulec. Integers Formalized Mathematics, 1(3):501-505, 1990.

[26] Wojciech A. Trybulec. Groups Formalized Mathematics, 1(5):821-827, 1990.

[27] Wojciech A. Trybulec. Vectors in real linear space. Formalized Mathematics, 1(2):291-296, 1990.

[28] Zinaida Trybulec. Properties of subsets Formalized Mathematics, 1(1):67-71, 1990.

[29] Tetsuya Tsunetou, Grzegorz Bancerek, and Yatsuka Nakamura. Zero-based finite sequences Formalized Mathematics, 9(4):825-829, 2001.

[30] Edmund Woronowicz. Relations and their basic properties. Formalized Mathematics, 1 (1):73-83, 1990. 